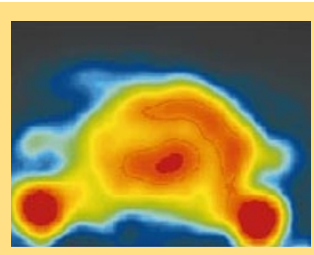

In this issue, Kornblum and coworkers report that a new technology designed for smallanimal imaging, microPET, can provide highresolution images of metabolic function in the conscious rat brain, and patterns of neuronal activity during seizures and physiological stimuli. They also demonstrate its usefulness for imaging neuronal depression and metabolic activity in the rat brain in a model of human epilepsy surgery. MicroPET may prove a useful tool for in vivo rodent models in the study and development of novel therapeutics for epilepsy, brain injury, and other disorders of the brain (see p. 655).

MS

\section{Healthier ketchup?}

Lycopene and $\beta$-carotene are major carotenoids that are not only essential for human health but also can apparently help reduce heart disease, certain cancers, and macular degeneration. Most people consume too few of these phytonutrients, and taking supplements may not be the answer, since other plant compounds are thought to exert a synergistic effect. To provide a better source of dietary carotenoids, Bramley and colleagues (p. 666) show that by engineering transgenic tomato plants to express a single bacterial gene for phytoene desaturase, they can produce fruit with three times as much $\beta$-carotene without affecting growth or development of the plant.

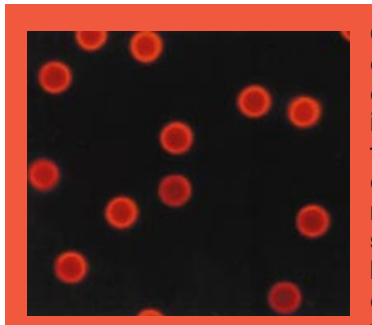
G proteincoupled receptors are important drug targets because of their roles in a wide spectrum of biological processes. CCR5 for example, is the principle co-receptor for transmission of HIV. However, efforts to screen for inhibitors of these receptors are often hampered by their tendency to unfold when extracted from their native membranes. On page 649, Mirzabekov and coworkers describe a method in which CCR5 is affixed to paramagnetic beads and surrounded with lipids, while retaining its native conformation. First they coated magnetic beads with streptavidin and an antibody that recognizes a tag on CCR5. They then used the beads to capture CCR5 from cell lysates, and the protein was reconstituted with detergent-solubilized lipids right on the bead. To demonstrate the utility of the approach, they used the beads to select CCR5-specific antibodies from a recombinant phage display library. N ND

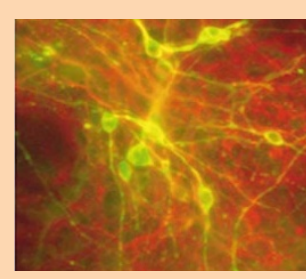

On page 675, McKay and colleagues describe a method for generating virtually unlimited supplies of dopaminergic and serotonergic neurons from mouse embryonic stem (ES) cells in vitro. By monitoring the expression of gene products from the midbrain and hindbrain regions of the brain that give rise to these specialized neurons, they identified the mitogenic and signaling molecules that promote their differentiation from ES progenitor cells. They expanded the population of these types of neurons and showed that they could indeed produce dopamine and serotonin. The ability to easily culture large populations of such functionally active neurons could provide an important tool for the study and treatment of neurodegenerative and psychiatric disorders.

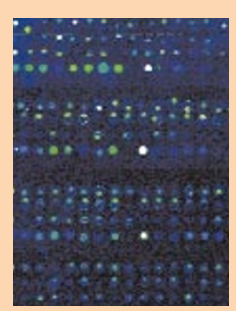

Microarray studies require substantial amounts of RNA to generate probes, especially when bacterial RNA is used for hybridization. On page 679, Talaat et al. describe a computer-based algorithm for predicting the minimal number of primers to specifically anneal to all genes in a given genome. They show that genome-directed primers (GDPs) are more sensitive and specific than random-primer probes when hybridized to an array of 960 Mycobacterium tuberculosis genes. The approach could be useful for genome-wide expression analysis and directed amplification of sequenced genomes.

\section{Review}

DNA sequence taken in isolation from a single organism reveals very little about gene function. In recent years, comparative genomics has provided new insights into our understanding of gene function and the evolutionary relationships that exist between genes. In functional genomics, computational analyses have been particularly useful in deducing putative functions for genes that can be tested by experimental analysis "wet" biology. Until recently, such methods for predicting function relied heavily on sequence comparison. On page 609, Galperin and Koonin discuss several newly developed computational approaches that significantly extend the predictive power of comparative genomics beyond sequence comparison. By analyzing the evolutionary relationships between protein families, protein and domain fusion comparisons in different species, gene adjacency in different genomes, and gene expression patterns, they argue computational genomics will provide deeper insights into functional interactions between proteins and information about specific functions.

\section{Retroviral vector for quiescent cells?}

Most retroviral vectors used for gene therapy are derived from C-type viruses, which cannot infect quiescent cells. Lentiviruses such as HIV-1, on the other hand, can infect quiescent cells, but concerns have been raised about their safety for human gene therapy. In this issue, Parveen et al. (see p. 623) describe how they developed an engineered retroviral vector that has the safety features of spleen necrosis virus (SNV), a C-type retroviral vector, and that can efficiently infect quiescent cells. Previous studies have suggested that HIV1 can infect nondividing cells with the help of a nuclear localization sequence (NLS) located in the matrix protein. The matrix proteins of SNV and HIV-1 are structurally similar, so the researchers tried mutating residues in the region of the SNV matrix protein analogous to the NLS in HIV-1. They found that by altering just two amino acids, they could produce a C-type retroviral vector capable of infecting growtharrested human $\mathrm{T}$ lymphocytes and quiescent primary monocyte-derived macrophages. In addition to its implications for gene therapy, the results support the notion that the NLS is sufficient to mediate the ability of HIV-1 to infect nondividing cells.

MS 